\title{
Estradiol Modulates Functional Brain Organization during the Menstrual Cycle: An Analysis of Interhemispheric Inhibition
}

\author{
Susanne Weis, ${ }^{1}$ Markus Hausmann, ${ }^{3}$ Barbara Stoffers, ${ }^{1}$ René Vohn, ${ }^{1}$ Thilo Kellermann, ${ }^{2}$ and Walter Sturm ${ }^{1}$ \\ ${ }^{1}$ Department of Neurology, Section Clinical Neuropsychology, and ${ }^{2}$ Department of Psychiatry and Psychotherapy, University Hospital Aachen, Rheinisch- \\ Westfälische Technische Hochschule Aachen University, 52074 Aachen, Germany, and 32Department of Psychology, Durham University, Durham DH1 3HP, \\ United Kingdom
}

According to the hypothesis of progesterone-mediated interhemispheric decoupling (Hausmann and Güntürkün, 2000), functional cerebral asymmetries (FCAs), which are stable in men and change during the menstrual cycle in women, are generated by interhemispheric inhibition of the dominant on the nondominant hemisphere. The change of lateralization during the menstrual cycle in women might indicate that sex hormones play an important role in modulating FCAs. We used functional magnetic resonance imaging to examine the role of estradiol in determining cyclic changes of interhemispheric inhibition. Women performed a word-matching task, while they were scanned twice during the cycle, once during the menstrual and once during the follicular phase. By use of a connectivity analysis we found that the inhibitory influence of left-hemispheric language areas on homotopic areas of the right hemisphere is strongest during the menses, resulting in a pronounced lateralization. During the follicular phase, due to rising estradiol levels, inhibition and thus functional cerebral asymmetries are reduced. These results reveal a powerful neuromodulatory action of estradiol on the dynamics of functional brain organization in the female brain. They may further contribute to the ongoing discussion of sex differences in brain function in that they help explain the dynamic part of functional brain organization in which the female differs from the male brain.

Key words: functional cerebral asymmetries; functional magnetic resonance imaging; menstrual cycle; sex differences; interhemispheric inhibition; connectivity

\section{Introduction}

The left hemisphere of the human brain is dominant for verbal tasks (e.g., Beaumont, 1982). A meta-analysis of imaging studies on sex differences in language lateralization (Kansaku and Kitazawa, 2001; Kitazawa and Kansaku, 2005) concluded that the degree of sex differences depends on the specific task used: differences were found when subjects performed phonological (Shaywitz et al., 1995; Pugh et al., 1996) or syntax tasks (Jaeger et al., 1998). No sex differences were observed for verbal tasks that were intrinsically semantic and applied exclusively to real words, not nonsense words (Buckner et al., 1995; Frost et al., 1999). Another

Received Sept. 13, 2008; revised 0ct. 7, 2008; accepted 0ct. 18, 2008.

This work was supported by grants from the Deutsche Forschungsgemeinschaft (STU-263/3-1 and HA 3285/4-1) and by the medical faculty of the University Aachen (START Grant 26/06). T.K. was supported by the Deutsche Forschungsgemeinschaft (DFG KF0 112/Ha 3202/2-2) and the Bundesministerium für Bildung und Forschung (BMBF 01GV0611). We thank the Functional Imaging Unit of the Interdisciplinary Centre for Clinical Research at the University Hospital Aachen, Germany, especially Georg Eder, for technical assistance during data acquisition, and Prof. J. Neulen (Clinic of Gynecologic Endocrinology and Reproductive Medicine, University Hospital Aachen, Aachen, Germany) for assistance with blood sample analysis. Further, we are grateful to Prof. Dr. A. D. Milner (Department of Psychology, Durham University, Durham, UK) for valuable comments and suggestions concerning earlier versions of this manuscript.

Correspondence should be addressed to Dr. Susanne Weis, Department of Neurology, Section Clinical Neuropsychology, University Hospital Aachen, Rheinisch-Westfälische Technische Hochschule Aachen University, Pauwellsstrasse 30, 52074 Aachen, Germany. E-mail: sweis@ukaachen.de.

DOI:10.1523/JNEUROSCI.4392-08.2008

Copyright $\odot 2008$ Society for Neuroscience ～0270-6474/08/2813401-10\$15.00/0 meta-analysis (Sommer et al., 2004) recently confirmed that on the whole no significant sex differences in language lateralization exist.

Functional cerebral asymmetries (FCAs) are stable in men while they change across the menstrual cycle in women (Hampson, 1990; Bibawi et al., 1995; Rode et al., 1995), suggesting that sex hormones such as progesterone and estradiol might modulate FCAs, but behavioral studies show inconsistent findings. Some studies found largest asymmetries during cycle phases high in steroid hormone concentration (Hampson, 1990; Bibawi et al., 1995), while others found maximal asymmetries during the low steroid menses (Rode et al., 1995; Mead and Hampson, 1996).

One study which did control for hormone concentration (Hausmann and Güntürkün, 2000) identified interactions between cycle phase and FCAs both in right- and left-hemispheric dominant tasks. The authors proposed that FCAs seem to be modulated by a global mechanism. Both hemispheres act as partially independent systems which each process stimuli simultaneously. Interhemispheric inhibition across the corpus callosum might be the key mechanism in coordinating the hemispheres and determining FCAs (Cook, 1984; Hoptman and Davidson, 1994; Chiarello and Maxfield, 1996), as it has been suggested before that the specialization of one hemisphere results from effective suppression of the other hemisphere (Kinsbourne, 1970). 
Hausmann and Güntürkün further assume that steroid hormones, especially progesterone, reduce FCAs, presumably through their glutamatergic and GABAergic effects which diminish corticocortical transmission (Hausmann and Güntürkün, 2000; Hausmann et al., 2002). Recent studies have shown that other hormones, especially estradiol, also influence FCAs (Hausmann, 2005; Holländer et al., 2005; Hausmann et al., 2006).

Few functional neuroimaging studies examined the neural correlates of lateralization changes across the menstrual cycle. One study found a general increase in brain activation with increasing estradiol levels (Dietrich et al., 2001), but no changes in FCAs between the menstrual and the follicular phase. Another functional magnetic resonance imaging (fMRI) study (Fernández et al., 2003) showed the extent of language-related activations to be significantly correlated with progesterone and less strongly with estradiol levels. While demonstrating that brain activation changes during the menstrual cycle, these studies did not provide any evidence concerning the underlying mechanisms.

We propose that FCAs are generated by an inhibitory influence of the dominant onto the nondominant hemisphere, which is modulated by sex hormones during the menstrual cycle (Hausmann and Güntürkün, 2000). An analysis of the localization of functional activations alone is not sufficient to depict the inhibitory influence of the dominant on the nondominant hemisphere. Thus, we set out to study the influence of estradiol on the change of FCAs across the menstrual cycle by means of a connectivity analysis.

\section{Materials and Methods}

Subjects. Fourteen right-handed, normally cycling women, who were not taking any hormonal contraceptives, were tested once during the menstrual phase (cycle day 1-3), and once during the follicular phase (cycle day 9-11). These two phases were chosen because they show the largest differences in estradiol levels. Progesterone and estradiol levels were assessed from blood samples taken directly after each experimental session to verify the cycle phase.

The mean age of the women was 26.83 years ( $\mathrm{SD}=4.98$ years; range 21-38 years). All women were consistent right-handers according to the Edinburgh Handedness Index (Oldfield, 1971) (mean EHI = 92.2, range 75-100) and had normal or corrected-to-normal vision. Women who had used hormonal contraceptives during the last 6 months were excluded.

Also, a control group of 14 right-handed men was examined twice at a time interval of $14 \mathrm{~d}$. All men had normal or corrected-to-normal vision; their age was 27.39 years ( $\mathrm{SD}=4.78$ years; range $24-39$ years). All men were consistent right-handers according to the Edinburgh Handedness Index (Oldfield, 1971) (mean EHI $=88.8$; range 80-100).

All subjects were native speakers of German.

Following approval by the Local Ethics Committee, all subjects gave their written informed consent according to the Declaration of Helsinki (1991). Both female and male subjects were paid for their participation.

Procedure. Before the experimental sessions, female subjects were informed about the general procedure and data about their menstrual cycle were collected. The women agreed to inform us on the first day of their next cycle to plan the dates for the experimental sessions. The individual length of each woman's menstrual cycle was taken into account when planning the appointments for the experiments. Every normally cycling woman was tested twice, once during the menstrual phase (cycle day 1-3) and once during the follicular phase (cycle day 9-11). Directly after each session a blood sample was collected from the female subjects. Progesterone and estradiol levels were assessed from the blood samples by Elektro-ChemiLumineszens ImmunoAssay (ECLIA) to evaluate the menstrual cycle phases. If necessary, the examination was repeated during the next cycle. To balance the design for the normally cycling women, the order of testing was randomized across subjects.

Men were tested twice, with a time interval of $14 \mathrm{~d}$. For statistical evaluation, a posteriori the first and second testing session was randomly assigned to experimental sessions 1 or 2 to avoid confounding effects of repeated measures.

To minimize circadian rhythm influences, every experimental session was performed at the same time of the day.

Materials and task. A pool of 160 German nouns was used for the lexical decision task, consisting of four to seven letters, selected for their high degree of abstractness (Baschek et al., 1977) to maximize the lefthemisphere advantage. Stimuli were delivered using the Presentation version 10.1 software package (Neurobehavioral Systems). In the MR scanner, participants viewed the stimuli via MRI compatible video goggles (VisuaStim XGA, Resonance Technology). In each experimental trial the subjects fixated a cross in the center of the monitor for a variable interstimulus interval (ISI), after which the first word appeared in the center of the screen for $185 \mathrm{~ms}$. One second later either the same or a different word (pseudorandomly alternated) was presented for $185 \mathrm{~ms}$ either to the left or to the right of the fixation cross while an empty frame appeared on the other side. The stimuli extended $4^{\circ}$ in the horizontal dimension with the inner edge being placed $2.2^{\circ}$ to the left or the right from the fixation cross. The experimental procedure was adapted from Hausmann and Güntürkün (Hausmann and Güntürkün, 1999). Subjects were instructed to fixate the center of the screen during the whole experiment and to indicate as quickly and as correctly as possible whether the laterally shown word was identical to the first word or not. The two words that were presented in a given trial had the same number of letters and the same first and last letter. Subjects responded with the index (same word) and middle finger (different word) of the left or right hand respectively.

Before entering the scanner, subjects were instructed not to move their head and body during the whole experiment and to fixate the fixation cross in the center of the screen. During fMRI scanning, subjects were presented with 20 blocks of eight trials each (two match trials in the left hemifield, two mismatch trials in the left hemifield, two match trials in the right hemifield, and two mismatch trials in the right hemifield in a pseudorandomized order). Before each block, the words "left-hand" or "right-hand" were presented for $10 \mathrm{~s}$ to indicate which hand had to be used for responding in the respective block. The interval from the onset of the first stimulus of one trial to the first stimulus of the next trial was jittered between 3970 and $4870 \mathrm{~ms}$, resulting in a mean ISI of $4420 \mathrm{~ms}$. Subjects were tested with parallel versions of the task, using the same stimuli, but in balanced order across the experimental sessions. Additionally, we collected data for two further tasks, which are not reported here.

fMRI data acquisition. All scans were performed on a 3 Tesla MRI scanner (Gyroscan Intera, Philips Medical Systems) using standard gradients and a circular polarized phase array head coil.

For each experimental session of each subject, one series of 476 functional volumes of $\mathrm{T}^{*}$-weighted axial EPI-scans including five initial dummy scans was acquired parallel to the $\mathrm{AC} / \mathrm{PC}$ line with the following parameters: number of slices (NS): 31 ; slice thickness (ST): $3.5 \mathrm{~mm}$; interslice gap (IG): $0.35 \mathrm{~mm}$; matrix size (MS): $64 \times 64$; field of view $(\mathrm{FOV}): 240 \times 240 \mathrm{~mm}$; echo time (TE): $30 \mathrm{~ms}$; repetition time (TR): $2 \mathrm{~s}$; flip angle (FA): $90^{\circ}$. During one of the experimental sessions, for each participant an anatomical scan was acquired using a high-resolution T1-weighted 3D-sequence (NS: 80; ST: $1 \mathrm{~mm}$; IG: $1 \mathrm{~mm}$; MS: $256 \times 256$; FOV: $256 \times 256 \mathrm{~mm}$; TE: $4.60 \mathrm{~ms}$; TR: $9900 \mathrm{~ms}$; FA $8^{\circ}$ ).

fMRI data analysis. MR images were analyzed using Statistical Parametric Mapping (SPM2; www.fil.ion.ucl.ac.uk) implemented in MATLAB 6.5 (Mathworks). After discarding the five dummy volumes, all images were realigned to the first image to correct for head movement. Unwarping was used to correct for the interaction of susceptibility artifacts and head movement. After realignment and unwarping, the signal measured in each slice was shifted in time relative to the acquisition time of the middle slice using a sinc interpolation to correct for their different acquisition times. Volumes were then normalized into standard stereotaxic anatomical MNI-space by using the transformation matrix calculated from the first EPI-scan of each subject and the EPI-template. The default settings for normalization in SPM2 with 16 nonlinear iterations and the standard EPI template supplied with SPM2 were used. Afterward, the normalized data with a resliced voxel size of $4 \mathrm{~mm} \times 4 \mathrm{~mm} \times$ 
$4 \mathrm{~mm}$ were smoothed with a $10 \mathrm{~mm}$ FWHM isotropic Gaussian kernel to accommodate intersubject variation in brain anatomy. The time series data were high-pass filtered with a high-pass cutoff of $1 / 128 \mathrm{~Hz}$. The first-order autocorrelations of the data were estimated and corrected for.

Four conditions were considered in the analyses, resulting from presentation to the right versus the left visual field and reaction with the right versus left hand. For the contrasts reported here, only the main effect of all conditions versus baseline was considered.

The expected hemodynamic response at stimulus onset was modeled by two response functions, a canonical hemodynamic response function (HRF) (Friston et al., 1998) and its temporal derivative. The temporal derivative was included in the model to account for the residual variance resulting from small temporal differences in the onset of the hemodynamic response, which is not explained by the canonical HRF alone. The functions were convolved with the event-train of stimulus onsets to create covariates in a general linear model. Subsequently, parameter estimates of the HRF regressor for each of the different conditions were calculated from the least mean squares fit of the model to the time series. Parameter estimates for the temporal derivative were not further considered in any contrast.

An SPM2 random-effects group analysis was performed by entering parameter estimates for all subjects into one-sample $t$ tests or paired $t$ tests, respectively. We report all functional activations at an uncorrected significance level of $p<0.005$.

On the basis of existing literature (Hinke et al., 1993; Fiez and Petersen, 1998; Dapretto and Bookheimer, 1999) we had an a priori hypothesis as to the most relevant activations for the word-matching task being located in the left inferior frontal gyrus (IFG). Still, we were interested in studying effects across the whole brain instead of restricting the search volume to predefined regions of interest. Therefore, we chose to employ a Monte-Carlo simulation of the brain volume to establish an appropriate voxel contiguity threshold (Slotnick et al., 2003). This correction has the advantage of higher sensitivity, while still correcting for multiple comparisons across the whole brain volume. Assuming an individual voxel type I error of $p<0.005$, a cluster extent of 32 contiguous resampled voxels was indicated as necessary to correct for multiple voxel comparisons across the whole brain at $p<0.01$ (based on 10,000 simulations).

The reported voxel coordinates of activation peaks were transformed from MNI space to Talairach and Tournoux atlas (Talairach and Tournoux, 1988) space by nonlinear transformations. The respective Matlab code can be found at http://imaging.mrc-cbu.cam.ac.uk/downloads/ MNI2tal/mni2tal.m. This was done to allow the use of the Talairach and Tournoux atlas (Talairach and Tournoux, 1988) to identify the anatomical brain regions for the activation peaks.

Psychophysiological interaction analysis. We calculated a psychophysiological interaction analysis (PPI) (Friston et al., 1997) to investigate the change of influence of the dominant left hemisphere on the nondominant right hemisphere. A seed region for the correlation was determined for each subject by selecting the individual left inferior frontal maximum closest to the maximum of the group analysis, which was located at Talairach and Tournoux coordinates (Talairach and Tournoux, 1988) $x=-36, y=23, z=-5$ (left IFG, Brodmann area [BA] 47). The seed region comprised a circular region with a radius of $10 \mathrm{~mm}$ around this voxel. The psychological variable was determined by the time course of the experiment, such that correlations during the experimental trial as opposed to baseline were calculated. Since we set out to identify the inhibitory influences of the dominant on the nondominant hemisphere, we identified those areas which showed a negative correlation with the seed region. Subsequently, the difference in connectivity between the menstrual and the follicular phase was identified using a paired $t$ test on the second level.

Same as for the functional activations, we used a Monte-Carlo simulation of the brain volume to establish an appropriate voxel contiguity threshold (Slotnick et al., 2003). For the main effects, i.e., inhibition during word-matching compared with baseline, assuming an individual voxel type I error of $p<0.01$, a cluster extent of 24 contiguous resampled voxels was indicated as necessary to correct for multiple voxel comparisons across the whole brain at $p<0.05$ (based on 10,000 simulations). For the difference contrasts, assuming an individual voxel type I error of $p<0.005$, a cluster extent of 32 contiguous resampled voxels was indicated as necessary to correct for multiple voxel comparisons across the whole brain at $p<0.01$ (based on 10,000 simulations).

To directly compare interhemispheric inhibition between male and female subjects, data from both tests of the men as well as the menstrual and the follicular phase in the women were entered into an ANOVA on the second level. Subsequently, the interaction between repeated tests and sex was computed. The results of this analysis are reported at an uncorrected threshold of $p<0.01$ with a minimal cluster size of 10 voxels.

As above, the reported voxel coordinates of activation peaks were transformed from MNI space to Talairach and Tournoux atlas (Talairach and Tournoux, 1988) space by nonlinear transformations.

Regression analysis. We computed a regression analysis between hormone levels and the mean parameter estimates in the cluster in right IFG which had been identified in the PPI analysis. These parameter estimates comprise a measure of the strength of the interhemispheric inhibition. Mean parameter estimates of the PPI analysis were extracted from the activation cluster in each individual female subject. Then a linear regression analysis was computed with estradiol and progesterone levels. To avoid confounding effects from repeated measures, regression analyses were computed for the menstrual and the follicular phase separately, resulting in two regression coefficients $r_{\mathrm{M}}$ and $r_{\mathrm{F}}$. Since the regression coefficient $r$ is not normally, but asymmetrically, distributed, regression coefficients need to be transformed to a normal distribution before the mean can be calculated. This was achieved by using Fisher's $z$-transformation:

$$
z(r)=1 / 2 \times \ln \left(\frac{1+r}{1-r}\right) .
$$

After averaging $z\left(r_{\mathrm{M}}\right)$ and $z\left(r_{\mathrm{F}}\right)$, the mean regression coefficient was calculated by the inverse transformation

$$
r(z)=\frac{e^{2 \times z}-1}{e^{2 \times z}+1} .
$$

\section{Results}

Hormone assays

The mean level of serum progesterone in our group of 14 women was $2.01(\mathrm{SEM}=0.25) \mathrm{nmol} / \mathrm{L}$ in the menstrual phase and 1.65 $(\mathrm{SEM}=0.17) \mathrm{nmol} / \mathrm{L}$ in the follicular phase. The mean level of serum estradiol of the women was $133.89($ SEM $=14.74) \mathrm{pmol} / \mathrm{L}$ in the menstrual phase and $350.14(\mathrm{SEM}=55.79) \mathrm{pmol} / \mathrm{L}$ in the follicular phase. A paired $t$ test revealed a significant cycle-phase difference in mean serum estradiol $\left(t_{(13)}=4.73, p<0.001\right)$, but not in progesterone levels $\left(t_{(13)}=2.12\right.$, n.s. $)$.

\section{Behavioral data}

The reaction time data and the number of correct responses of the female and male group were subjected to $2 \times 2$ linear mixedeffects ANOVAs including a general correlation structure to better account for dependencies between observations. Visual hemifield (left, right) and cycle phase (menstrual phase, follicular phase) respectively test session (test 1 , test 2) were treated as within-subject factors, while subjects were treated as random effects. To accommodate dependencies between mean and SDs for binomially distributed data, the number of correct responses was arcsin-transformed.

\section{Female subjects}

The behavioral data for the women are given in Table 1. For reaction times the mixed-effects ANOVA identified a significant effect of hemifield $\left(F_{(1,39)}=4.56, p<0.04\right)$. The interaction of hemifield and cycle phase approached significance $\left(F_{(1,39)}=3.85\right.$, $p=0.057)$. The effect of cycle phase was not significant $\left(F_{(1,39)}=\right.$ 0.92 , n.s.) 
Table 1. Behavioral data for the normally cycling women

\begin{tabular}{lll}
\hline & Menses & Follicular phase \\
\hline RT & & \\
$\quad$ Left visual field (SEM) & $930.36 \mathrm{~ms}(46.81 \mathrm{~ms})$ & $896.49 \mathrm{~ms}$ (45.97 ms) \\
$\quad$ Right visual field (SEM) & $880.18 \mathrm{~ms}(40.58 \mathrm{~ms})$ & $873.37 \mathrm{~ms}$ (44.34 ms) \\
$\%$ correct responses & & \\
$\quad$ Left visual field (SEM) & $67.76(3.86)$ & $69.83(3.25)$ \\
Right visual field (SEM) & $82.67(4.00)$ & $80.71(3.65)$ \\
\hline
\end{tabular}

Reaction times and percentage of correct responses for items presented to the right and the left visual hemifield during the menstrual and the follicular phase.

Table 2. Behavioral data for the male group

\begin{tabular}{lll}
\hline & Exp. session 1 & Exp. session 2 \\
\hline RT & & \\
$\quad$ Left visual field (SEM) & $784.43 \mathrm{~ms}(41.52 \mathrm{~ms})$ & $795.06 \mathrm{~ms}$ (45.27 ms) \\
$\quad$ Right visual field (SEM) & $756.20 \mathrm{~ms}(33.31 \mathrm{~ms})$ & $775.64 \mathrm{~ms}$ (42.55 ms) \\
$\begin{array}{l}\text { \% correct responses } \\
\text { Left visual field (SEM) }\end{array}$ & $69.64(2.95)$ & $69.29(3.71)$ \\
$\quad$ Right visual field (SEM) & $82.14(3.01)$ & $78.30(4.90)$ \\
\hline
\end{tabular}

Reaction times and percentage of correct responses for items presented to the right and the left visual hemifield during experimental session 1 and experimental session 2.

To further explore the nature of the interaction, alphaadjusted post hoc paired $t$ tests (Bonferroni corrected) were computed comparing reaction times for the left and the right hemifield during each of the cycle phases. The analysis revealed a significant right visual field/left hemisphere advantage in reaction time only during the menstrual phase $\left(t_{(13)}=2.82, p<\right.$ $0.014)$ while during the follicular phase, the reaction times between the left and right visual field were not significantly different $\left(t_{(13)}=2.35\right.$, n.s. $)$.

The mixed-effects ANOVA for the number of correct responses only identified a significant main effect of visual hemifield $\left(F_{(1,39)}=57.11, p<0.0001\right)$. Neither the main effect for cycle phase nor the interaction between cycle phase and visual hemifield approached significance $\left(\right.$ all $F_{(1,39)}<1.72$, n.s.).

\section{Male subjects}

The behavioral data for the men are given in Table 2. For the reaction time data of the men the mixed-effects ANOVA identified a significant effect of hemifield $\left(F_{(1,39)}=4.87, p<0.04\right)$ only, again indicating a right visual field/left hemisphere advantage for the word-matching task. Neither the main effect of test session nor the interaction between hemifield and test session approached significance (all $F_{(1,39)}<0.56$, n.s.).

Also in the respective analysis for the number of correct responses, a significant main effect of visual hemifield $\left(F_{(1,39)}=\right.$ $29.74, p<0.001$ ) was found. Again, neither the main effect of test session nor the interaction between hemifield and test session approached significance, (all $F_{(1,39)}<0.80$, n.s.).

\section{Sex differences in accuracy and reaction time}

The reaction time data of the men and the women were subjected to a $2 \times 2 \times 2$ linear mixed-effects ANOVA, with visual hemifield (left, right) and measurement (time point a and b) as fixed within-subjects factors and sex (male, female) as fixed between subjects factor. Subjects were treated as random effects. The levels of the factor measurement refer to the different time points. For the female group, however, the levels distinguished two different cycle phases whereas in the male group the two time points have been randomly assigned to time point a or $\mathrm{b}$. The analyses identified a significant effect of $\operatorname{sex}\left(F_{(1,26)}=4.42, p<0.05\right)$ and visual hemifield $\left(F_{(1,78)}=9.31, p<0.004\right)$. The interaction of measure- ment and visual hemifield approached significance $\left(F_{(1,78)}=\right.$ 3.87, $p=0.0527)$. No further main effect or interactions were significant (all $F<1.01$, n.s.).

The respective analysis for the number of correct responses identified a significant effect of visual hemifield $\left(F_{(1,78)}=73.52\right.$, $p<0.0001)$ and measurement $\left(F_{(1,78)}=5.11, p<0.03\right)$. Also, the interaction of visual hemifield and measurement approached significance $\left(F_{(1,78)}=3.77, p<0.056\right)$. No further main effect or interaction approached significance (all $F<1.08$, n.s.).

\section{Imaging results}

Both in the female and in the male group the central activation for the word-matching task as opposed to baseline was located in the left IFG (BA 47). In women, during the follicular phase this was the only significant activation, while during the menstrual phase, the activation was somewhat more extended and included parts of the left middle frontal gyrus (BA 45), as well as the cingulate cortex extending into bilateral superior and medial frontal gyri (BA $6,8,32$ ). The activations are shown projected onto a standard brain in Figure 1, $A$ and $B$, and all maxima are reported in Table 3.

When directly comparing activation for the word-matching task between the menstrual and the follicular phase, no significant difference was found in either direction.

For the male group, activations during both experimental sessions comprised a network including left frontal cortex, centered in the IFG (BA 45, 47), extending into the middle frontal gyrus (BA 9, 46) and the insula (BA 13), as well as a medial frontal activation including bilateral cingulate gyri and superior and medial frontal gyri (BA 6, 8, 32). There was also a smaller activation of a right temporo-parieto-occipital cluster, including the superior and middle temporal gyri, the cuneus and precuneus, the supramarginal and angular gyri as well as the inferior and superior parietal lobules (BA 7, 19, 39, 40).

No significant difference between the two experimental sessions could be identified.

The activations are shown projected onto a standard brain in Figure 1, $C$ and $D$, and all maxima are reported in Table 3.

\section{Results of the PPI analysis}

In the women during the menstrual phase, the PPI analysis identified one cluster located in the right frontal cortex. This cluster spans parts of the right medial, middle, inferior, and superior frontal gyri, extending into the anterior cingulate (BA 9, 10, 32, 46). During the follicular phase no significant inhibitory influence of the left IFG was identified. The results of the PPI analysis for the female group are reported in Table 4 and shown in Figure 2.

When comparing the inhibitory influence of the left IFG during the menstrual and the follicular phases, we observed a significantly stronger inhibitory influence on the right IFG during the menstrual phase (Fig. 2), extending into the right middle frontal gyrus (BA 11, 47). No brain region was found that was significantly more strongly inhibited by the left IFG during the follicular phase.

For the male group there was no significant change in interhemispheric inhibition between the two experimental sessions. The results of the PPI analysis for the male group are reported in Table 4.

The interaction analysis between cycle phase and sex identified all brain regions in which interhemispheric connectivity does change between cycle phases in women, but remains stable in men across repeated tests. This interaction was significant in the 

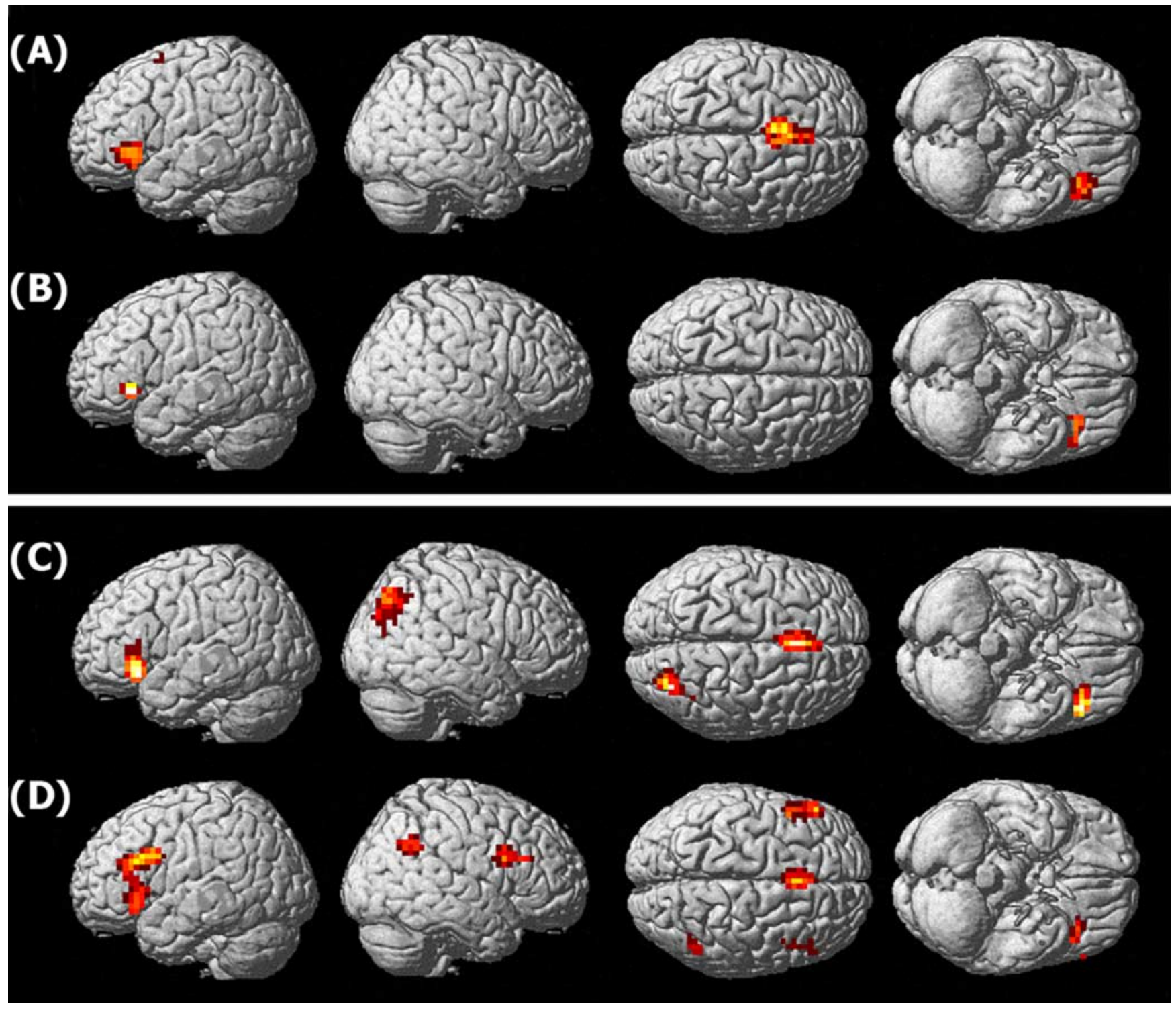

Figure 1. Functional activations of the normally cycling women and the male group. $A$, Female subjects: regions activated for word-matching compared with baseline during the menstrual phase $(p<0.01$, corrected for multiple comparisons). $\boldsymbol{B}$, Female subjects: regions activated for word-matching compared with baseline during the follicular phase ( $p<0.01$, corrected for multiple comparisons). C, Male subjects: regions activated for word-matching compared with baseline during experimental session 1 ( $p<0.01$, corrected for multiple comparisons). $\boldsymbol{D}$, Male subjects: regions activated for word-matching compared with baseline during experimental session 2 ( $p<0.01$, corrected for multiple comparisons).

right hemisphere homotope of the left IFG. The parameter estimates, which are depicted in Figure 3, provide a measure for the degree of interhemispheric inhibition in this region.

Further brain regions identified in the analysis are listed in Table 4.

Results of the regression analysis

Estradiol correlated negatively with the strength of the interhemispheric inhibition both during the menstrual phase $(r=-0.546$, $p<0.05)$ and the follicular phase $(r=-0.614, p<0.02)$. The mean correlation coefficient for both phases was $r=-0.581$, which is significant at $p<0.03$. It should be noted that the relationship between estradiol level and strength of interhemispheric inhibition might not be linear for the range of estradiol levels measured in the menstrual phase (Fig. 4).

In contrast, progesterone did not show any significant correlation with the strength of the interhemispheric inhibition in either the menstrual ( $r=-0.054$, n.s. $)$ or the follicular phase $(r=$ -0.072 , n.s.).

\section{Discussion}

The present study was conducted to study dynamic changes in functional brain organization in women, which, among other factors, contribute to the understanding of functional differences between the male and female brain. Even though we used a verbal task here, the implications of our results might not be restricted to the domain of language processing, since functional asymmetries are a fundamental principle of functional brain organization which applies not only to language but to the majority of higher cognitive functions. The few existing functional brain imaging studies on changes in lateralization during the menstrual cycle have focused on specific cognitive tasks and the change of the respective brain activations during the cycle (Dietrich et al., 2001; Fernández et al., 2003). The present study, however, focuses more 
Table 3. Functional activations of the female and the male subjects

\begin{tabular}{|c|c|c|c|c|c|c|}
\hline & \multirow[b]{2}{*}{ BA } & \multicolumn{3}{|c|}{ Coordinates } & \multirow[b]{2}{*}{$Z$ value } & \multirow[b]{2}{*}{ No. voxels } \\
\hline & & $x$ & $y$ & $z$ & & \\
\hline \multicolumn{7}{|l|}{ 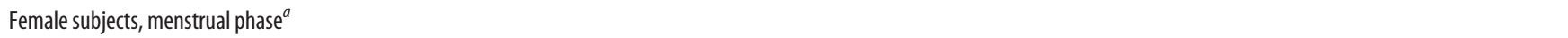 } \\
\hline Left superior frontal gyrus & 6 & -4 & 7 & 55 & 4.05 & 123 \\
\hline Left inferior frontal gyrus & 47 & -36 & 27 & -1 & 3.85 & 84 \\
\hline \multicolumn{7}{|l|}{ Female subjects, follicular phase ${ }^{a}$} \\
\hline Left inferior frontal gyrus & 47 & -36 & 27 & -5 & 3.31 & 36 \\
\hline \multicolumn{7}{|c|}{ Male subjects, experimental session $1^{a}$} \\
\hline Left inferior frontal gyrus & 47 & -32 & 27 & 2 & 4.03 & 112 \\
\hline Left cingulate gyrus & 32 & -4 & 22 & 43 & 4.50 & 86 \\
\hline Right superior parietal lobule & 7 & 28 & -68 & 44 & 3.57 & 96 \\
\hline \multicolumn{7}{|c|}{ Male subjects, experimental session $2^{a}$} \\
\hline Left medial frontal gyrus & 8 & 0 & 22 & 43 & 3.98 & 57 \\
\hline Left middle frontal gyrus & 9 & -44 & 13 & 25 & 3.90 & 179 \\
\hline Right inferior frontal gyrus & 9 & 44 & 13 & 21 & 4.06 & 60 \\
\hline Right angular gyrus & 39 & 32 & -61 & 33 & 3.63 & 63 \\
\hline
\end{tabular}

Activation peaks with their localization, significance level and the size of the respective activation cluster (number of voxels). Coordinates are listed in Talairach and Tournoux (1988) atlas space. BA is the Brodmann area nearest to the coordinate and should be considered approximate.

${ }^{a}$ Word-matching $>$ baseline $(p<0.01$, corrected)

Table 4. Results of the PPI analysis of the female and the male subjects

\begin{tabular}{|c|c|c|c|c|c|c|}
\hline & \multirow[b]{2}{*}{ BA } & \multicolumn{3}{|c|}{ Coordinates } & \multirow[b]{2}{*}{ Zvalue } & \multirow[b]{2}{*}{ No. voxels } \\
\hline & & $x$ & $y$ & $Z$ & & \\
\hline \multicolumn{7}{|l|}{ Females, menstrual phase ${ }^{a}$} \\
\hline Right middle frontal gyrus & 11 & 40 & 44 & -8 & 2.88 & 121 \\
\hline \multicolumn{7}{|c|}{ Females, menstrual phase $>$ follicular phase ${ }^{b}$} \\
\hline Right inferior frontal gyrus & 47 & 44 & 34 & -19 & 3.54 & 43 \\
\hline \multicolumn{7}{|l|}{ Males, experimental session $1^{a}$} \\
\hline Left middle frontal gyrus & 10 & -28 & 43 & 9 & 2.64 & 39 \\
\hline Left paracentral lobule & 5 & -20 & -44 & 46 & 2.86 & 96 \\
\hline Left insula & 13 & -40 & -22 & 23 & 2.63 & 38 \\
\hline Right insula & 13 & 44 & -18 & 23 & 3.11 & 58 \\
\hline Right precuneus & 7 & 16 & -40 & 46 & 2.82 & 36 \\
\hline Right lingual gyrus & 19 & 32 & -62 & -4 & 2.70 & 26 \\
\hline \multicolumn{7}{|l|}{ Males, experimental session $2^{a}$} \\
\hline Left superior frontal gyrus & 9 & 0 & 56 & 30 & 3.5 & 54 \\
\hline Left posterior cingulate & 29 & -8 & -46 & 10 & 4.33 & 2436 \\
\hline Left inferior parietal lobule & 40 & -59 & -37 & -46 & 3.46 & 52 \\
\hline Left paracentral lobule & 4 & 0 & -36 & 64 & 3.74 & 347 \\
\hline Left middle occipital gyrus & 19 & -40 & -81 & 15 & 3.36 & 45 \\
\hline Right superior temporal gyrus & 38 & 48 & 11 & -11 & 3.64 & 80 \\
\hline Right middle frontal gyrus & 46 & 51 & 32 & 24 & 3.23 & 35 \\
\hline \multicolumn{7}{|c|}{ Interaction between sex and measurement ${ }^{c}$} \\
\hline Right angular gyrus & 39 & 40 & -76 & 30 & 3.07 & 18 \\
\hline Right inferior frontal gyrus & 47 & 55 & 15 & -4 & 2.77 & 12 \\
\hline Left cuneus & 19 & -16 & -88 & 27 & 2.76 & 16 \\
\hline Right superior temporal gyrus & 22 & 44 & -24 & -6 & 2.74 & 10 \\
\hline Left parahippocampal gyrus & 36 & -20 & -25 & -26 & 2.69 & 28 \\
\hline Right cuneus & 31 & 16 & -73 & 18 & 2.62 & 21 \\
\hline Left lingual gyrus & 18 & -20 & -74 & 0 & 2.54 & 13 \\
\hline
\end{tabular}

Activation peaks with their localization, significance level, and the size of the respective activation cluster (number of voxels). Coordinates are listed in Talairach and Tournoux (1988) atlas space. BA is the Brodmann area nearest to the coordinate and should be considered approximate.

${ }^{a} p<0.05$, corrected.

${ }^{b} p<0.01$, corrected.

${ }^{c} p<0.01$, minimal cluster size 10 voxels.

on general principles of hormone related changes in functional brain activation, which should generally apply to a variety of lateralized cognitive functions and might thus help to explain the dynamics of the functional brain organization in women as well as contribute to the on-going discussion of sex differences.

A verbal task was exemplary chosen to study these mechanisms, since this task was used in several previous behavioral experiments and always revealed a robust right visual half-field advantage which reflects a superiority of the left hemisphere
(Rode et al., 1995; Hausmann et al., 2002). Furthermore, the word-matching task has been shown to be sensitive to hormonal fluctuations across the menstrual cycle (Rode et al., 1995; Hausmann et al., 2002). Finally, previous fMRI studies have suggested that this task can be assumed to be clearly localized to the left inferior frontal gyrus (Hinke et al., 1993; Fiez and Petersen, 1998; Dapretto and Bookheimer, 1999). All these assumptions were confirmed by our data.

Based on the hypothesis of hormone-mediated interhemi- 

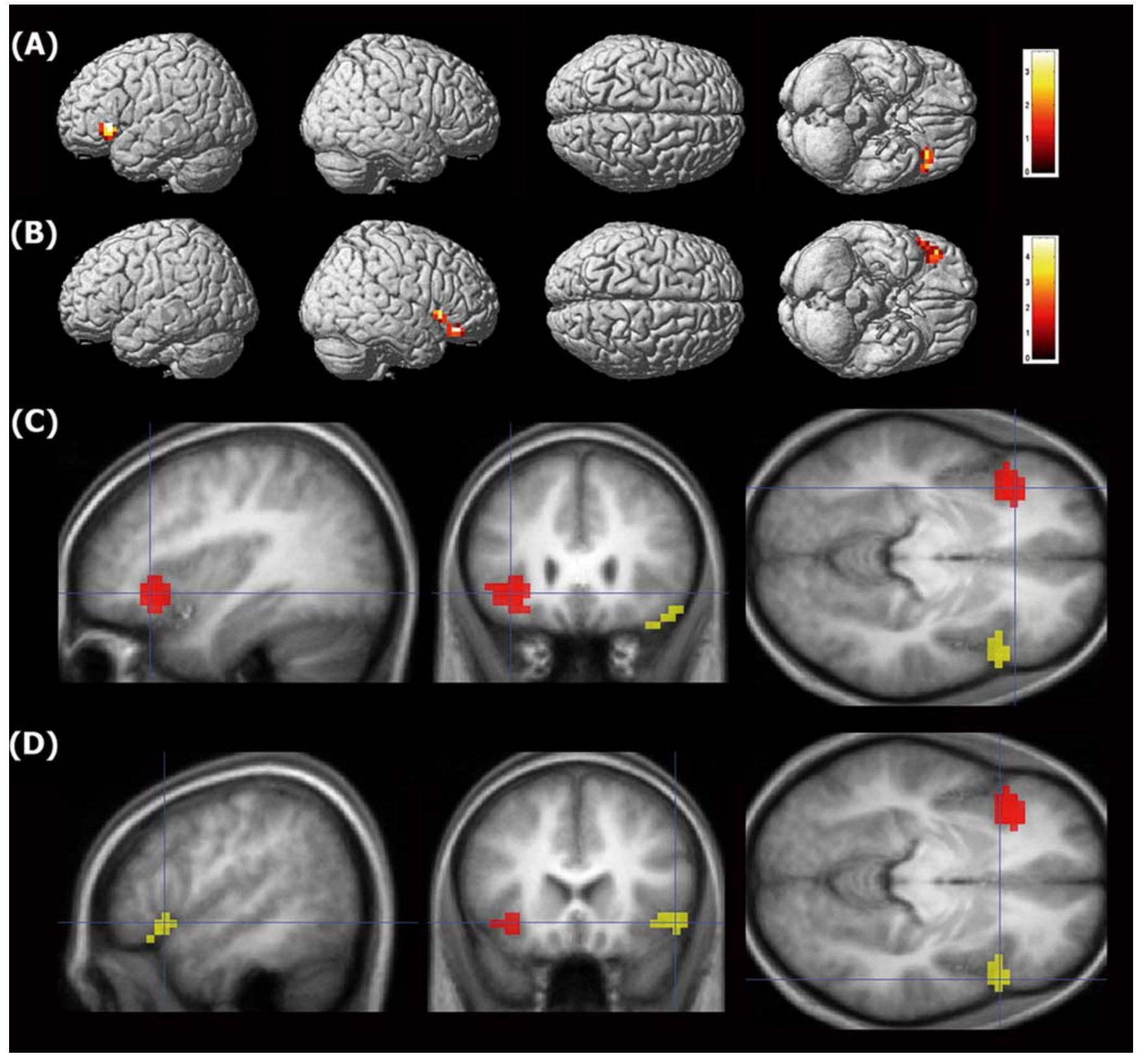

Figure 2. Functional imaging and connectivity analysis. The most significant activation during word-matching compared with rest across cycle phases in the female group was located in left IFG (A). A seed region was defined in each female subject as a sphere around the individual local maximum located closest to the maximum of the group activation. Then a PPI analysis was used to identify those brain regions on which the seed region exerts a significantly stronger inhibitory influence for word-matching during the menstrual compared with the follicular phase. The only such region was located in right IFG $(\boldsymbol{B})$. The activation maps (conjunction analysis at $p<0.01$, corrected for multiple comparisons in $\boldsymbol{A}$, PPI analysis at $p<0.01$, corrected for multiple comparisons in $\boldsymbol{B}$ ) are shown overlaid onto a canonical brain rendered in three dimensions. The anatomical location of the most significant activation in left IFG (shown in red) and the area in right IFG which is inhibited more significantly during the follicular phase (shown in yellow) is shown overlaid onto the mean high-resolution T1 scan of the female group at the maximum of the left IFG activation ( $\boldsymbol{C}$ and the maximum of the inhibited area (D).

spheric decoupling (Hausmann and Güntürkün, 2000), which claims interhemispheric inhibition to be the central process in generating FCAs, we used a connectivity analysis to delineate the inhibitory influence of the dominant on the nondominant hemisphere, instead of focusing on the task-specific activations and their change during the menstrual cycle.

For the verbal task used in the present study, we identified the left IFG (Fig. 1) as the central functional area, both in female and in male subjects. This brain region has been shown to be selectively involved with the processing of semantic aspects of verbal information, word reading and internal speech (Hinke et al., 1993; Fiez and Petersen, 1998; Dapretto and Bookheimer, 1999).
It is interesting to note that no significant differences between cycle phases were found when comparing the activation of the left IFG during word-matching between the menstrual and the follicular phase.

The comparison of the reaction times between sexes revealed that FCAs were more pronounced in the female subjects, while for the accuracy data no sex differences were found. These findings are in line with the results of several meta-analyses showing that for semantic language tasks no reliable sex differences exist (Kansaku and Kitazawa, 2001; Sommer et al., 2004; Kitazawa and Kansaku, 2005).

Even though there was no significant difference in activation 
of the left hemispheric language area between cycle phases, the behavioral data of the female group showed a trend toward an interaction between left hemisphere advantage and cycle phase. Further, a significant advantage of the left hemisphere for the word-matching task was identified during the menstrual, but not during the follicular phase. Such an interaction was absent in the male control group, in which left IFG activation also remained stable across repeated tests.

The PPI analysis identified a significant change in functional connectivity between the IFG of the dominant and the nondominant hemisphere between the menstrual and the follicular phase in women. It is interesting to note that the area which is affected by this change of left IFG inhibitory influence in relation with estradiol level is located in almost the exactly homotopic area of the right hemisphere. These areas correspond to those found by Shaywitz and coworkers to be more bilaterally activated in female compared with male subjects during a verbal task (Shaywitz et al., 1995). As the interaction analysis shows, the change of interhemispheric inhibition in right IFG is specifically related to different cycle phases in the female sub-
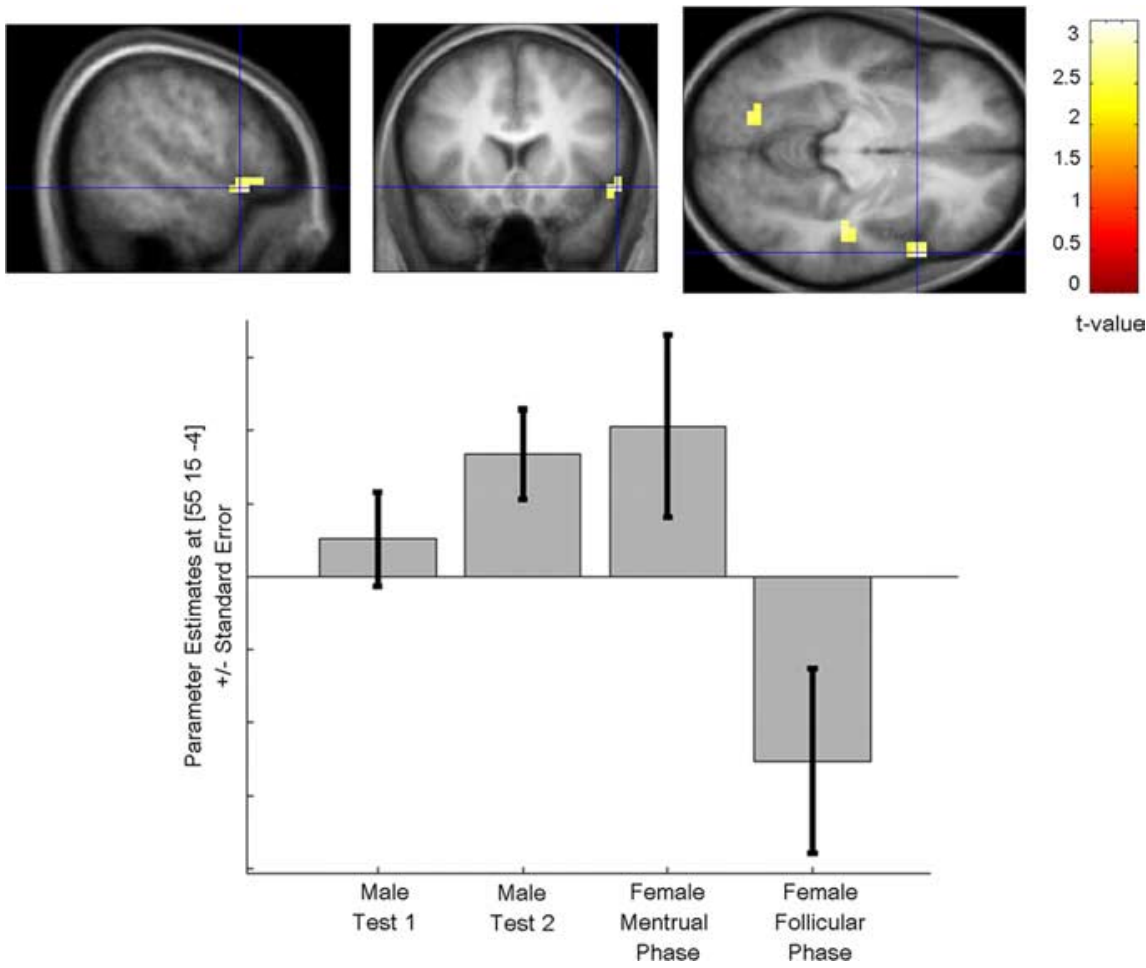

t-value

Figure 3. Sex interaction analysis. The interaction effect of the PPI analysis between repeated tests and sexes at $p<0.01$ uncorrected, at a minimal cluster size of 10 voxels is shown overlaid onto the mean high-resolution T1 scan of the male and female group at the maximum of the right IFG activation. Parameter estimates of in the maximum of the cluster are depicted. Parameter estimates are given in arbitrary units (a.u.). jects, while in males, interhemispheric inhibition remains stable over time. The parameter estimates clearly show that during both tests in men as well as during the menstrual phase in women the left IFG exerts an inhibitory influence on the homotopic area of the right hemisphere. During the follicular phase in the women, no interhemispheric inhibition is found.

The results of our connectivity analysis contribute to the understanding of differences of functional brain organization between the sexes: in women, we have shown interhemispheric inhibition to change significantly between the menstrual and the follicular phase, resulting in significantly more pronounced FCAs in the menstrual phase. In men, on the other hand, interhemispheric inhibition was shown to remain stable between tests. We propose that these mechanisms are not specific for the verbal task used here, but can be assumed to be universally involved with all lateralized higher cognitive functions.

Our results clearly support the model of Hausmann and Güntürkün (Hausmann and Güntürkün, 2000) whereby interhemispheric interactions form the key component in generating FCAs. Also, they are in accordance with a number of studies which used electrophysiological approaches, particularly TMS (Ferbert et al., 1992; Mochizuki et al., 2004; Lee et al., 2007) to confirm the role of the corpus callosum in interhemispheric inhibition.

Our data further reveal an important role of estradiol in addition to the established role of progesterone in modulating interhemispheric inhibition across the menstrual cycle. In corroboration of existing results, we identified most pronounced FCAs during the menstrual phase, the cycle phase lowest in gonadal steroid hormones levels. We were also able to show, however, that not only rising progesterone levels during the luteal phase do result in diminished interhemispheric interaction and thus reduced FCAs (Hausmann and Güntürkün, 2000; Hausmann et al.,
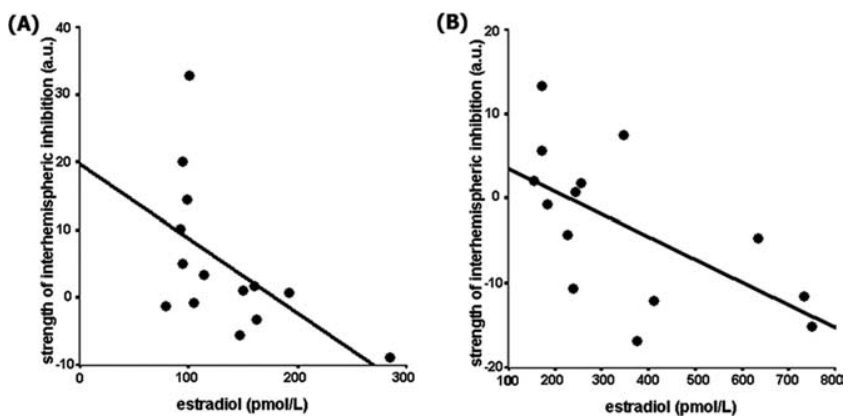

Figure 4. Correlation of estradiol and strength of interhemispheric inhibition. The correlation of estradiol level and strength of interhemispheric inhibition in right IFG is significant both during the menstrual ( $r=-0.546, p<0.05$, shown in $A$ ) and the follicular $(r=-0.614, p<$ 0.02 , shown in $\boldsymbol{B}$ ) phase. As a measure for the strength of interhemispheric inhibition we used the size of the parameter estimates, which were extracted from the right inferior frontal cluster identified in the PPI analysis. Parameter estimates are given in arbitrary units (a.u.).

2002), but similar mechanism occur during the follicular phase with rising estradiol levels.

We did not find any correlation between interhemispheric inhibition and progesterone levels during the menstrual and the follicular phase. Since there is only a small variance in progesterone levels during these cycle phases, this does not necessarily imply an independence of interhemispheric inhibition and progesterone. For estradiol, however, we were able to identify a significant correlation with the strength of interhemispheric inhibition. Thus, FCAs are directly related to estradiol level in normally cycling women. While there is a strong inhibition of the dominant onto the nondominant hemisphere resulting in pronounced FCAs during the low-estradiol menstrual phase, the interhemi- 
spheric inhibition is reduced in connection with rising estradiol levels during the follicular phase.

Obviously, fMRI cannot directly elucidate the neurobiological basis of the effects we found. From the findings reported here, it can only be stated that changes in interhemispheric inhibition occur in correlation with fluctuating estradiol levels. Based on fMRI data alone, however, it is impossible to infer the underlying neurobiological mechanisms and the causal relationship between fluctuations in estradiol levels and cycle-phase-related changes in BOLD response.

Hausmann and Güntürkün (Hausmann and Güntürkün, 2000) originally assumed that most callosal fibers arise from excitatory and glutamatergic neurons which mostly terminate on pyramidal neurons in the contralateral hemisphere which then activate GABAergic interneurons. Although callosal fibers are mainly excitatory (glutamate-mediated), the net-effect on homotopic areas of the contralateral hemisphere is a short (GABAA mediated) and long interhemispheric inhibition (GABAB mediated) (Innocenti, 1980; Voigt et al., 1988; Peters et al., 1990; Hughes and Peters, 1992; Conti and Manzoni, 1994), inducing a widespread inhibition of homotopic regions of the contralateral hemisphere.

Receptors of various transmitter systems are fundamentally modulated by gonadal steroid hormones such as estradiol and progesterone (Majewska et al., 1986; Smith et al., 1987a,b, 1988; Smith, 1991). Especially, high progesterone levels in the luteal phase might reduce the callosal activation by its agonistic and antagonistic effects on glutamate and GABA receptors, respectively. Although estradiol and progesterone have partly opposite neuromodulatory properties on glutamate and GABA receptors, a previous TMS study (Hausmann et al., 2006) found that estradiol and progesterone have almost identical effects on transcallosal inhibition during the follicular and luteal phase, respectively. This might suggest that either estradiol and progesterone affect the cascade of neuromodulatory processes similarly but at different sites, or both estradiol and progesterone are only indirectly involved but are precursors or metabolites of the active steroid hormone.

In any case, our results corroborate recent findings suggesting the need to extend the hypothesis of progesterone-mediated interhemispheric decoupling (Hausmann and Güntürkün, 2000) to include the influence of estradiol (Hausmann, 2005; Holländer et al., 2005).

These findings correspond with the results of a recent study which used transcranial magnetic stimulation (TMS) to study changes in transcallosal inhibition (TCI) during the menstrual cycle (Hausmann et al., 2006). The authors found a negative correlation between both TCI and progesterone levels during the luteal phase and TCI and estradiol levels during the follicular phase. Just as the data presented here, these findings suggest that a similar inhibitory effect is modulated by different sex hormones during different phases of the cycle.

Also, our results are in accordance with a number of recent behavioral studies that have identified an important role of estradiol in modulating FCAs. One study examining the "attentional blink" found an association of both progesterone and estradiol with reduced FCAs during the midluteal phase. Estradiol, however, was identified as the key factor (Holländer et al., 2005), with progesterone playing only a minor role. Further evidence for the important role of estradiol on FCAs comes from studies on transsexuals. One study showed that male-to-female transsexuals showed a rapid shift from male-typical FCAs to a more female- typical symmetric pattern after treatment with estradiol (Van Goozen et al., 1995).

In conclusion, we used functional neuroimaging to directly depict the mechanism behind the change of FCAs across the menstrual cycle by use of a connectivity analysis. Our data point to estradiol as one of the key factors for the modulation of interhemispheric inhibition and thereby for changes of FCAs between the menstrual and the follicular phase.

Our findings may not be specific for the verbal task used here but might delineate a more general mechanism of hormonemediated changes in functional brain organization. The same mechanisms are supposedly involved with any lateralized cognitive function, both for right and left hemisphere dominant tasks. To verify this assumption, further imaging research, especially using right hemisphere dominant task is needed. If, as we assume, the same mechanisms can be shown for such tasks, these results would confirm previous studies revealing striking neuromodulatory properties of gonadal steroid hormones on the dynamics of functional brain organization in the female brain.

Moreover, the present study might constructively contribute to the debate about sex differences in FCAs. A recent metaanalysis of imaging studies focusing on sex differences in lateralization (Sommer et al., 2004) quantifies sex differences as relatively small to not existent. However, it should be noted that almost none of these studies controlled for cycle phase and/or underlying sex hormone levels. The present study clearly supports the necessity to control for these variables as confounding factors.

It is generally agreed that sex differences are based both on anatomical differences and on short term dynamical changes in functional brain organization. While no conclusions can be drawn from our findings concerning neuroanatomical differences between the male and female brain, they clearly show that differences in functional brain organization exist between the sexes. If studies would take those confounding factors into account, we would probably get a much clearer picture about sex differences in the brain.

\section{References}

Baschek IL, Bredenkamp J, Oehrle B, Wittich W (1977) Bestimmung der Bildhaftigkeit, Konkretheit und der Bedeutungshaltigkeit von 800 Substantiven. Zeitschrift fuer experimentelle und angewandte Psychologie 24:353-396.

Beaumont JG (1982) Studies with verbal stimuli. In: Divided visual field studies of cerebral organization. (Beaumont JG, ed), pp 58-86. London: Academic.

Bibawi D, Cherry B, Hellige JB (1995) Fluctuations of perceptual asymmetry across time in women and men: effects related to the menstrual cycle. Neuropsychologia 33:131-138.

Buckner RL, Raichle ME, Petersen SE (1995) Dissociation of human prefrontal cortex areas across different speech production tasks and gender groups. J Neurophysiol 74:2163-2173.

Chiarello C, Maxfield L (1996) Varieties of interhemispheric inhibition, or how to keep a good hemisphere down. Brain Cogn 30:81-108.

Conti F, Manzoni T (1994) The neurotransmitters and postsynaptic actions of callosally projecting neurons. Behav Brain Res 64:37-53.

Cook ND (1984) Callosal inhibition: the key to the brain code. Behav Sci 29:98-110.

Dapretto M, Bookheimer SY (1999) Form and content: dissociating syntax and semantics in sentence comprehension. Neuron 24:427-432.

Dietrich T, Krings T, Neulen J, Willmes K, Erberich S, Thron A, Sturm W (2001) Effects of blood estrogen level on cortical activation patterns during cognitive activation as measured by functional MRI. Neuroimage 13:425-432.

Ferbert A, Priori A, Rothwell JC, Day BL, Colebatch JG, Marsden CD (1992) Interhemispheric inhibition of the human motor cortex. J Physiol 453:525-546. 
Fernández G, Weis S, Stoffel-Wagner B, Tendolkar I, Reuber M, Beyenburg S, Klaver P, Fell J, de Greiff A, Ruhlmann J, Reul J, Elger CE (2003) Menstrual cycle-dependent neural plasticity in the adult human brain is hormone, task, and region specific. J Neurosci 23:3790-3795.

Fiez JA, Petersen SE (1998) Neuroimaging studies of word reading. Proc Natl Acad Sci U S A 95:914-921.

Friston KJ, Buechel C, Fink GR, Morris J, Rolls E, Dolan RJ (1997) Psychophysiological and modulatory interactions in neuroimaging. Neuroimage 6:218-229.

Friston KJ, Fletcher P, Josephs O, Holmes A, Rugg MD, Turner R (1998) Event-related fMRI: characterizing differential responses. Neuroimage 7:30-40.

Frost JA, Binder JR, Springer JA, Hammeke TA, Bellgowan PS, Rao SM, Cox RW (1999) Language processing is strongly left lateralized in both sexes. Evidence from functional MRI. Brain 122:199-208.

Hampson E (1990) Variations in sex-related cognitive abilities across the menstrual cycle. Brain Cogn 14:26-43.

Hausmann M (2005) Hemispheric asymmetry in spatial attention across the menstrual cycle. Neuropsychologia 43:1559-1567.

Hausmann M, Güntürkün O (1999) Sex differences in functional cerebral asymmetries in a repeated measures design. Brain Cogn 41:263-275.

Hausmann M, Güntürkün O (2000) Steroid fluctuations modify functional cerebral asymmetries: the hypothesis of progesterone-mediated interhemispheric decoupling. Neuropsychologia 38:1362-1374.

Hausmann M, Becker C, Gather U, Güntürkün O (2002) Functional cerebral asymmetries during the menstrual cycle: a cross-sectional and longitudinal analysis. Neuropsychologia 40:808-816.

Hausmann M, Tegenthoff M, Sänger J, Janssen F, Güntürkün O, Schwenkreis P (2006) Transcallosal inhibition across the menstrual cycle: a TMS study. Clin Neurophysiol 117:26-32.

Hinke RM, Hu X, Stillman AE, Kim SG, Merkle H, Salmi R, Ugurbil K (1993) Functional magnetic resonance imaging of Broca's area during internal speech. Neuroreport 4:675-678.

Holländer A, Hausmann M, Hamm JP, Corballis MC (2005) Sex hormonal modulation of hemispheric asymmetries in the attentional blink. J Int Neuropsychol Soc 11:263-272.

Hoptman MJ, Davidson RJ (1994) How and why do the two cerebral hemispheres interact? Psychol Bull 116:195-219.

Hughes CM, Peters A (1992) Symmetric synapses formed by callosal afferents in rat visual cortex. Brain Res 583:271-278.

Innocenti GM (1980) The primary visual pathway through the corpus callosum: morphological and functional aspects in the cat. Arch Ital Biol 118:124-188.

Jaeger JJ, Lockwood AH, Van Valin RD Jr, Kemmerer DL, Murphy BW, Wack DS (1998) Sex differences in brain regions activated by grammatical and reading tasks. Neuroreport 9:2803-2807.

Kansaku K, Kitazawa S (2001) Imaging studies on sex differences in the lateralization of language. Neurosci Res 41:333-337.

Kinsbourne M (1970) The cerebral basis of lateral asymmetries in attention. Acta Psychol (Amst) 33:193-201.

Kitazawa S, Kansaku K (2005) Sex difference in language lateralization may be task-dependent. Brain 128:E30.
Lee H, Gunraj C, Chen R (2007) The effects of inhibitory and facilitatory intracortical circuits on interhemispheric inhibition in the human motor cortex. J Physiol 580:1021-1032.

Majewska MD, Harrison NL, Schwartz RD, Barker JL, Paul SM (1986) Steroid hormone metabolites are barbiturate-like modulators of the GABA receptor. Science 232:1004-1007.

Mead LA, Hampson E (1996) Asymmetric effects of ovarian hormones on hemispheric activity: evidence from dichotic and tachistoscopic tests. Neuropsychology 10:578-587.

Mochizuki H, Huang YZ, Rothwell JC (2004) Interhemispheric interaction between human dorsal premotor and contralateral primary motor cortex. J Physiol 561:331-338.

Oldfield RC (1971) The assessment and analysis of handedness: the Edinburgh inventory. Neuropsychologia 9:97-113.

Peters A, Payne BR, Josephson K (1990) Transcallosal non-pyramidal cell projections from visual cortex in the cat. J Comp Neurol 302:124-142.

Pugh KR, Shaywitz BA, Shaywitz SE, Constable RT, Skudlarski P, Fulbright RK, Bronen RA, Shankweiler DP, Katz L, Fletcher JM, Gore JC (1996) Cerebral organization of component processes in reading. Brain 119:1221-1238.

Rode C, Wagner M, Güntürkün O (1995) Menstrual cycle affects functional cerebral asymmetries. Neuropsychologia 33:855-865.

Shaywitz BA, Shaywitz SE, Pugh KR, Constable RT, Skudlarski P, Fulbright RK, Bronen RA, Fletcher JM, Shankweiler DP, Katz L (1995) Sex differences in the functional organization of the brain for language. Nature 373:607-609.

Slotnick SD, Moo LR, Segal JB, Hart J Jr (2003) Distinct prefrontal cortex activity associated with item memory and source memory for visual shapes. Brain Res Cogn Brain Res 17:75-82.

Smith SS (1991) Progesterone administration attenuates excitatory amino acid responses of cerebellar Purkinje cells. Neuroscience 42:309-320.

Smith SS, Waterhouse BD, Chapin JK, Woodward DJ (1987a) Progesterone alters GABA and glutamate responsiveness: a possible mechanism for its anxiolytic action. Brain Res 400:353-359.

Smith SS, Waterhouse BD, Woodward DJ (1987b) Locally applied progesterone metabolites alter neuronal responsiveness in the cerebellum. Brain Res Bull 18:739-747.

Smith SS, Waterhouse BD, Woodward DJ (1988) Locally applied estrogens potentiate glutamate-evoked excitation of cerebellar Purkinje cells. Brain Res 475:272-282.

Sommer IE, Aleman A, Bouma A, Kahn RS (2004) Do women really have more bilateral language representation than men? A meta-analysis of functional imaging studies. Brain 127:1845-1852.

Talairach J, Tournoux P (1988) Co-planar stereotaxic atlas of the human brain. Stuttgart, Germany: Thieme.

Van Goozen SH, Cohen-Kettenis PT, Gooren LJ, Frijda NH, Van de Poll NE (1995) Gender differences in behaviour: activating effects of cross-sex hormones. Psychoneuroendocrinology 20:343-363.

Voigt T, LeVay S, Stamnes MA (1988) Morphological and immunocytochemical observations on the visual callosal projections in the cat. J Comp Neurol 272:450-460. 